Supporting Information

\title{
Deep Exciton Self-Trapping Cu-Based Perovskite Nanocrystals for Optoelectronic Applications
}

Thanh-Hai Le, ${ }^{1}$ Sanghyuck Lee, ${ }^{2}$ Hyemi Jo, ${ }^{2}$ Minjin Kim, ${ }^{2}$ Jisun Lee, ${ }^{2}$ Mincheol Chang,${ }^{1,2, *}$ Hyeonseok Yoon ${ }^{1,2, *}$

${ }^{1}$ Alan G. MacDiarmid Energy Research Institute \& School of Polymer Science and Engineering, Chonnam National University, 77 Yongbong-ro, Buk-gu, Gwangju 61186, South Korea

${ }^{2}$ Department of Polymer Engineering, Graduate School, Chonnam National University 77 Yongbong-ro, Buk-gu, Gwangju 61186, South Korea

*E-mail: hyoon@chonnam.ac.kr; mchang35@chonnam.ac.kr 


\section{EXPERIMENTAL SECTION}

\section{Materials.}

Caesium carbonate $\left(\mathrm{Cs}_{2} \mathrm{CO}_{3}, 99.9 \%\right)$, copper (I) bromide $(\mathrm{CuBr}, 99.9 \%)$, copper (I) iodide (CuI, 99.9\%), copper (I) chloride ( $\mathrm{CuCl}, 99.9 \%$ ), octadecene (ODE, 90\%), , oleylamine (OLA, 70\%), anhydrous hexane (99.9\%), and anhydrous ethyl acetate (EtOAc, 99.8\%) were purchased from Aldrich. Hexylamine (HLA, >99\%), nonanoic acid (NA, >98\%), nonylamine (NLA, $>99 \%$ ), and oleic acid (OA, 90\%) were purchased from Tokyo Chemical Industry.

Preparation of caesium carboxylate salts.

$1.4 \mathrm{mmol} \mathrm{Cs}_{2} \mathrm{CO}_{3}, 22.5 \mathrm{~mL}$ ODE, and $1.5 \mathrm{~mL}$ of OA were placed in a flask and degassed at $120^{\circ} \mathrm{C}$ for $10 \mathrm{~min}$. The mixture was heated to $150^{\circ} \mathrm{C}$ under the inner condition until a clear solution was obtained. The transparent solution containing caesium oleate was stored for further use. Similarly, OA was simply replaced by HA or NA to prepare caesium hexanoat or caesium nonylate.

\section{Synthesis of copper halide PNCs.}

Copper halide PNCs were prepared via the following procedure: $5.73 \mathrm{mg} \mathrm{CuBr}$ and $10 \mathrm{~mL}$ ODE were mixed in a three-neck reactor and heated to $100^{\circ} \mathrm{C}$ under a vacuum environment. Then, an equimolar ratio (1.6:1.6 mmol:mmol) of a pair of - C6-C6 (HA and HLA), C9-C9 (NA and NLA), C18-C18 (OA and OLA), C6-C9 (HA and NLA), or C9-C18 (NA and OLA) - was added into the $\mathrm{CuBr} / \mathrm{ODE}$ solution under nitrogen. The reaction mixture was further heated to $120^{\circ} \mathrm{C}$ until a clear blue solution was achieved (Figure S1). Then, the reactor temperature was reduced to $70^{\circ} \mathrm{C}$, followed by quick injection of $3 \mathrm{~mL}$ of the corresponding $\mathrm{Cs}-$ carboxylate salt as prepared above. After $25 \mathrm{~s}$, the reactor was cooled by an ice-water bath. The synthesized solution was directly transferred to centrifuge tubes and subjected to centrifugation. The resulting precipitate was redispersed in anhydrous hexane for further use.

\section{Characterization.}

Transmission electron microscopy (TEM) images and selected area electron diffraction (SAED) patterns were acquired by a JEOL JEM-2100F. Optical absorption spectra were collected using 
an Optizen 2120UV spectrophotometer (Mecasys, Korea). PL spectra were measured using a Hitachi F-4500 fluorescence spectrophotometer (Japan) under $270 \mathrm{~nm}$ excitation. Powder X-ray diffraction (XRD) analysis was carried out on a Rigaku X-ray diffractometer (D/MAX-2500, Japan) with $\mathrm{Cu} \mathrm{K} \alpha$ radiation $\left(\lambda=1.5418 \AA\right.$ ). The average size (D) of $\mathrm{Cs}_{3} \mathrm{Cu}_{2} \mathrm{Br}_{5}$ nanocrystals were calculated based on Scherrer's equation as,

$$
D=\frac{0.9 \times \lambda}{d \times \cos \theta}
$$

where, $\lambda$ is the X-ray wavelength (1.5418 $\AA), d$ is the full width at half maximum intensity of the most intense peak (in radian). The average size was estimated to be 100, 32, and $23 \mathrm{~nm}$ for C6, C9, and $\mathrm{C} 18$ ligand capped $\mathrm{Cs}_{3} \mathrm{Cu}_{2} \mathrm{Br}_{5}$ nanocrystals.

\section{Numerical calculation.}

Geometry optimizations of ligands were conducted using the B3LYP functional combined with 6-31G(d) split-valence basis sets in gaussian09. The pseudopotential plane-wave method with the Perdew-Burke-Ernzerhof (PBE) functional was used for the electronic property calculations, implemented in quantum expresso software. $\mathrm{Cs}_{3} \mathrm{Cu}_{2} \mathrm{X}_{5}$ has a base-centered orthorhombic structure with the Pnma (\#62) space group. Experimental lattice parameters (a=10.42 $\AA, b=$ $11.83 \AA, \mathrm{c}=14.59 \AA$ for $\mathrm{Cs}_{3} \mathrm{Cu}_{2} \mathrm{I}_{5} ; \mathrm{a}=9.85 \AA, \mathrm{b}=11.11 \AA, \mathrm{c}=13.85 \AA$ for $\mathrm{Cs}_{3} \mathrm{Cu}_{2} \mathrm{Br}_{5} ; \mathrm{a}=9.47$ $\AA, \mathrm{b}=10.63 \AA, \mathrm{c}=13.3 \AA$ for $\mathrm{Cs}_{3} \mathrm{Cu}_{2} \mathrm{Cl}_{5}$ ) were used. The kinetic energy cutoff of $25 \mathrm{Ry}$ ( $225 \mathrm{Ry}$ for the charge density) and the plane-wave basis were used for all calculations. Electronic structures and density of states (DOSs) were calculated using the PBE functional, which includes the exchange-correlation energy functional within the generalized gradient approximation (GGA). 


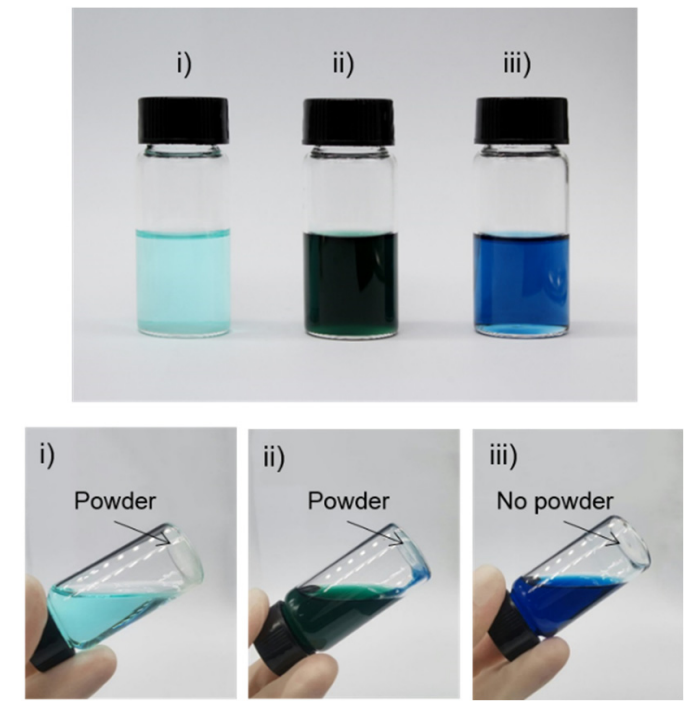

Figure S1. Optical images of CuBr solution dissolved by (i) acid-, (ii) amine-, and (iii) a pair of acid- and amine-based hydrocarbon ligands. 


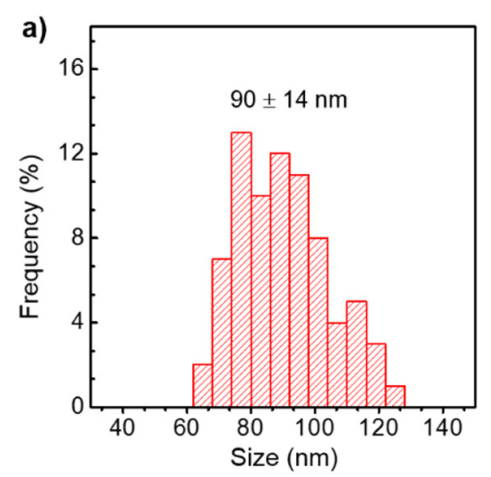

b)

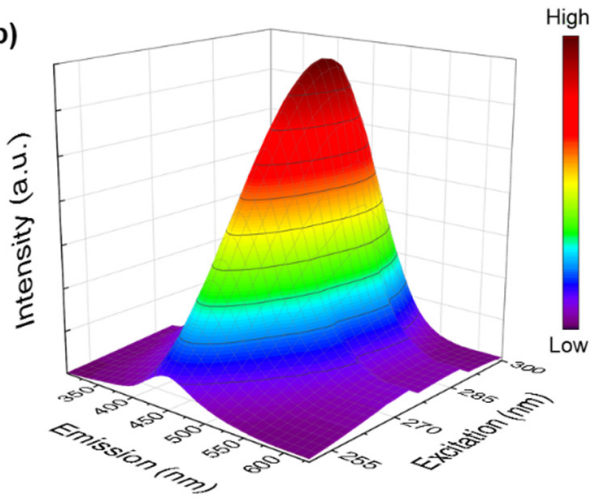

Figure S2. CNPs: a) Size distribution and b) PL intensity as a function of excitation and emission wavelengths. 

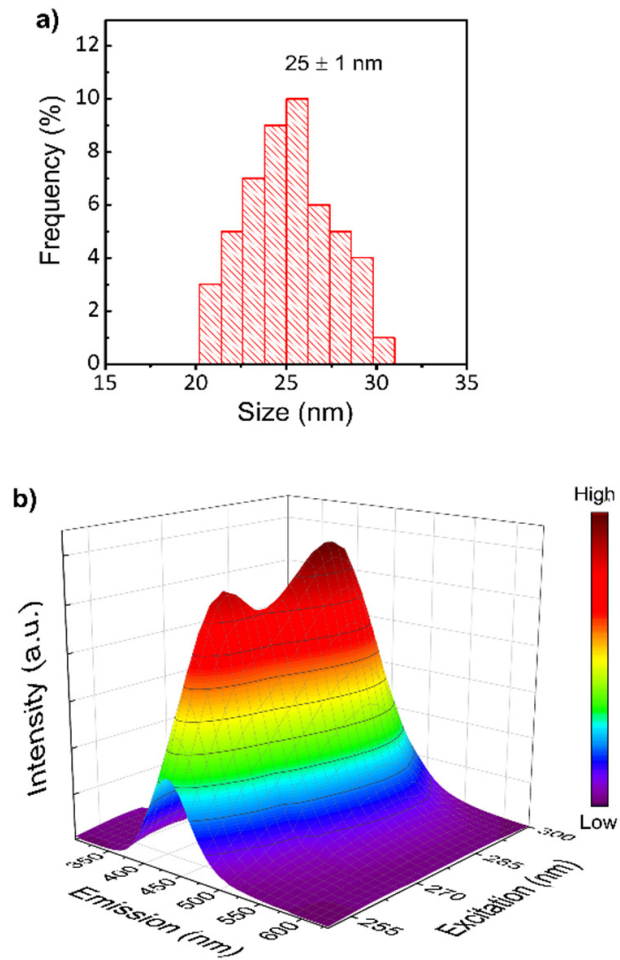

Figure S3. CNCs: a) Size distribution and b) PL intensity as a function of excitation and emission wavelengths. 

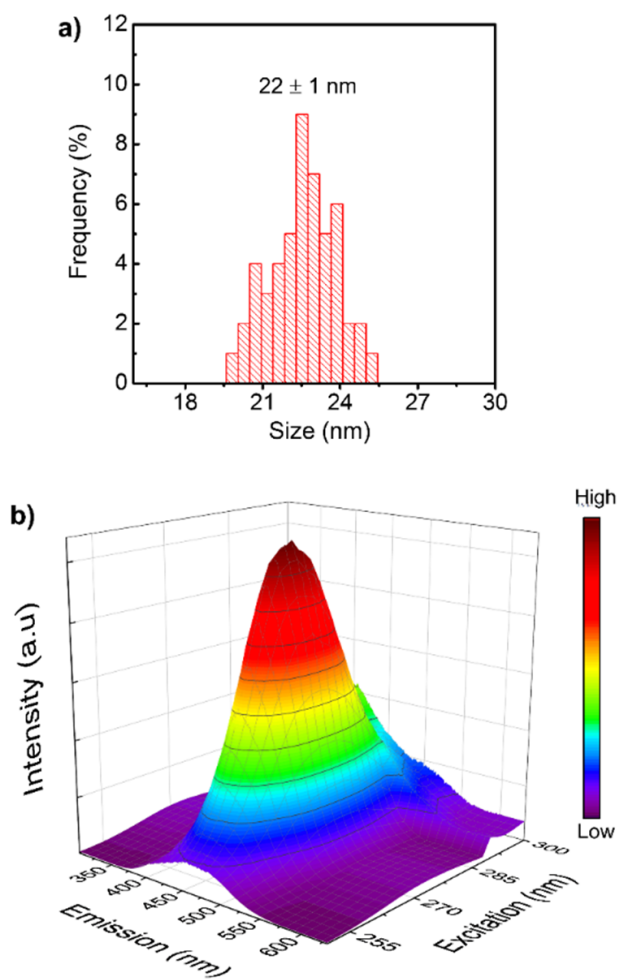

Figure S4. CNSs: a) Size distribution and b) PL intensity as a function of excitation and emission wavelengths. 
a)

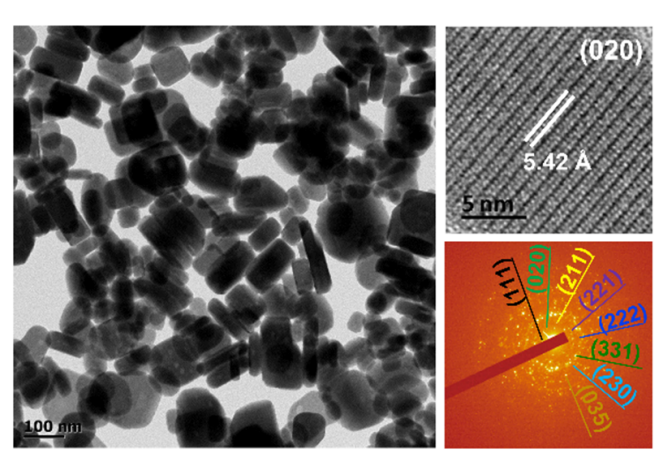

b)
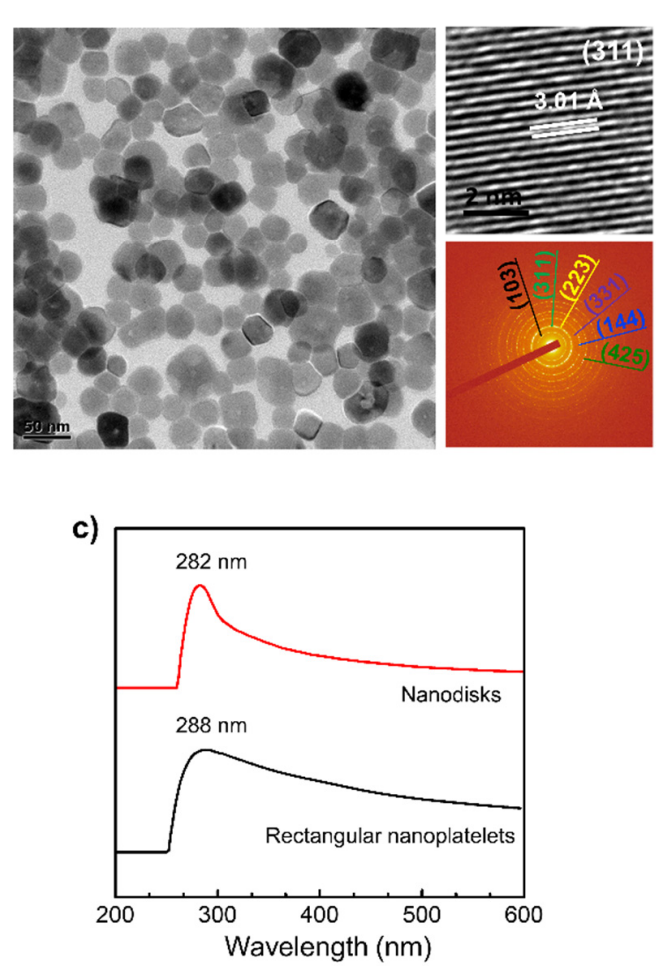

Figure S5. $\mathrm{Cs}_{3} \mathrm{Cu}_{2} \mathrm{Br}_{5}$ nanocrystals synthesized using two combinations of ligands with different chain lengths. TEM images, SAED patterns, and HRTEM images of $\mathrm{Cs}_{3} \mathrm{Cu}_{2} \mathrm{Br}_{5}$ rectangular nanoplates (CNRs) and $\mathrm{Cs}_{3} \mathrm{Cu}_{2} \mathrm{Br} 5$ nanodisks (CNDs) synthesized using (a) HA (C6)/NLA (C9) and (b) NA (C9)/OLA (C18), respectively; and (c) Optical absorption of CNRs (black) and CNDs (red). 


\section{Effect of mixing different chain-length ligands}

To further investigate the effect of the ligands on morphology, combinations of two different ligands with various chain lengths were employed: HA/NLA (C6/C9) and NA/OLA (C9/C18). Figure S5a presents a TEM image of $\mathrm{Cs}_{3} \mathrm{Cu}_{2} \mathrm{Br}_{5}$ nanocrystals prepared with $\mathrm{HA} / \mathrm{NLA}$. The image reveals large rectangular nanoplates (comparable to the size of CNPs) with nonuniform size and shape. Notably, the short ligand HA was insufficient for control of nanocrystal growth even in the presence of the longer ligand NLA. Changing the ligands to NA/OLA resulted in the formation of nanodisks with a diameter of $32.4 \pm 5.1 \mathrm{~nm}$ (Figure S5b and Figure S6a). The typical thickness of the CNDs was approximately $9.2 \mathrm{~nm}$, as determined from line profiles in atomic force microscope (AFM) images (Figure S6b). The clear lattice fringes and SAED patterns of the CNRs and CNDs also demonstrate their high degree of crystallinity, in which the interplanar distances of $5.42 \AA$ (CNPs) and $3.01 \AA$ (CNDs) correspond to the (020) and (311) crystallographic planes, respectively, of orthorhombic $\mathrm{Cs}_{3} \mathrm{Cu}_{2} \mathrm{Br}_{5}$. The absorption spectra of CNRs and CNDs reveal major peaks at $288 \mathrm{~nm}(4.30 \mathrm{eV})$ and $282 \mathrm{~nm}(4.36 \mathrm{eV})$, which are located near the middle of the peaks of CNPs-CNCs and CNCs-CNSs, respectively. This result strongly suggests a clear correlation between the chain length of the ligands and the morphology of the nanocrystals: while the use of short-chain ligands leads to the formation of large, nonuniform nanocrystals, the use of long-chain ligands result in smaller nanospheres with controlled sizes. With increasing ligand chain length from $\mathrm{C} 6$ to $\mathrm{C} 18$, the ligand becomes more hydrophobic, thereby providing higher steric hindrance effect to slow down the nucleationgrowth process. ${ }^{1}$ Additionally, in contrast with C6 and C9 ligands, C18 ligands have a kind structure with a double bond at the 9th carbon position (Figure S7a), which can provide more free volume on the surface of nuclei precursor. These characteristics of the ligands lead to the 
isotropic or anisotropic growth, which may account for the different shapes of the nanocrystals.

In addition, with increasing ligand chain length from $\mathrm{C} 6$ to $\mathrm{C} 18$, the ligand becomes more hydrophobic, which may provide more colloidal stability in a hydrophobic medium for preventing undesirable destructive aggregation (Figure S7b). ${ }^{1}$ 
a)

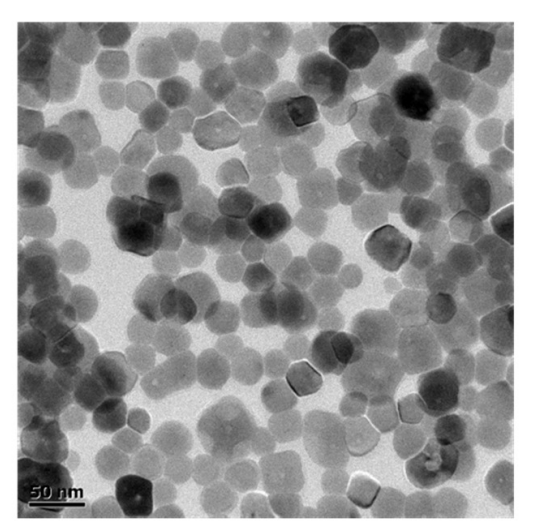

b)

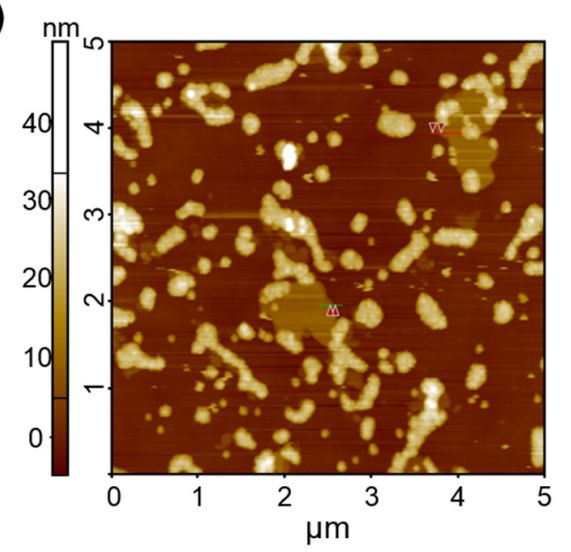

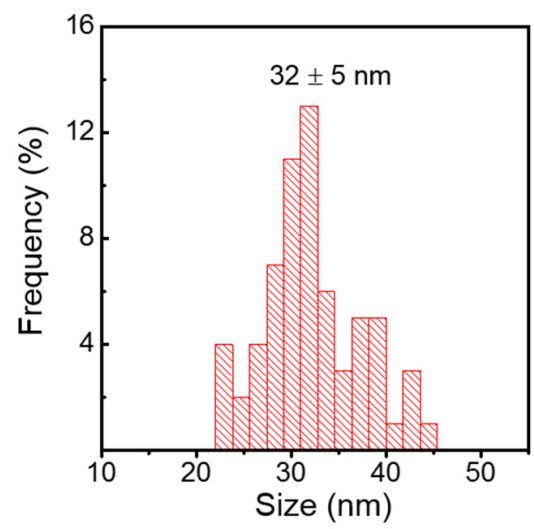

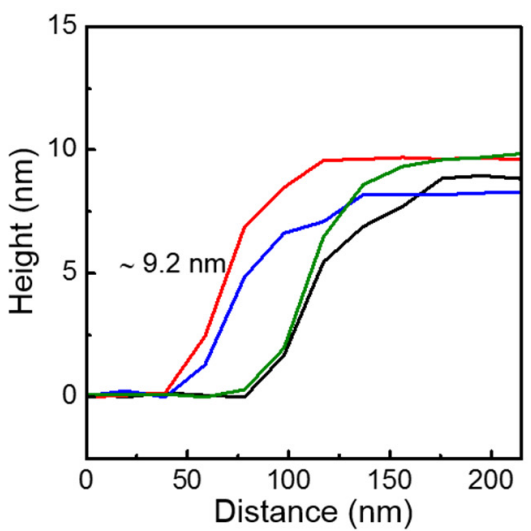

Figure S6. a) TEM image and histogram showing the CND size distribution. b) AFM topography micrograph of CNDs with height profiles for the selected lines. 
a)

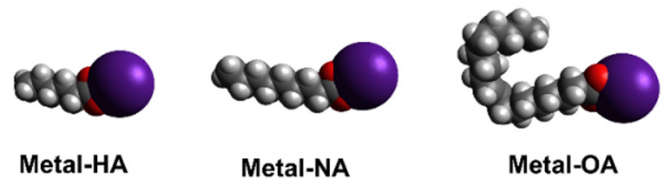

b)

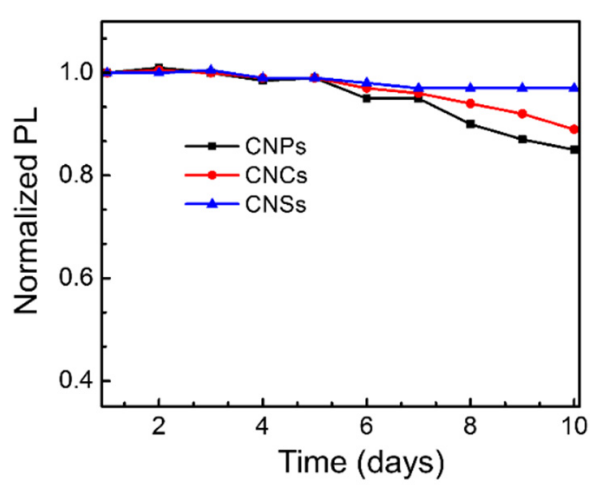

Figure S7. a) Optimized chemical structures of ligand capped metal ion using B3LYP functional with LANL2DZ basis set. b) PL retention of CNPs, CNCs, and CNSs over storage in a dark cabinet. 


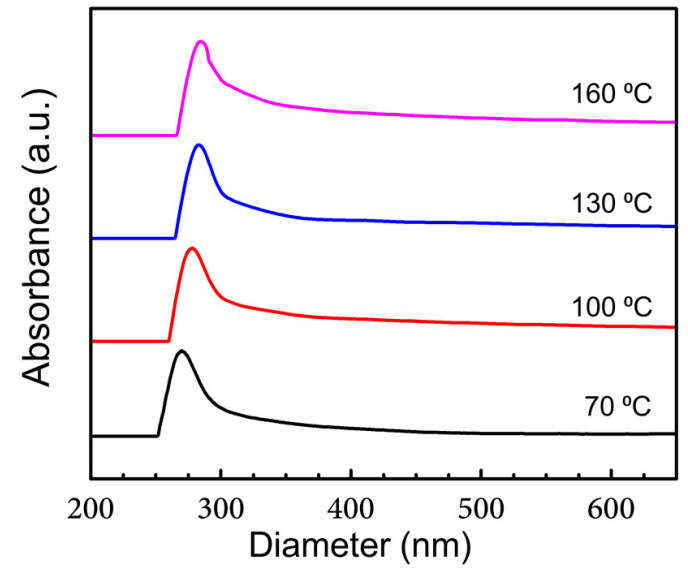

Figure S8. UV-vis absorption of the nanocrystals synthesized at $70^{\circ} \mathrm{C}, 100^{\circ} \mathrm{C}, 130^{\circ} \mathrm{C}$, and $160^{\circ} \mathrm{C}$. 
a)

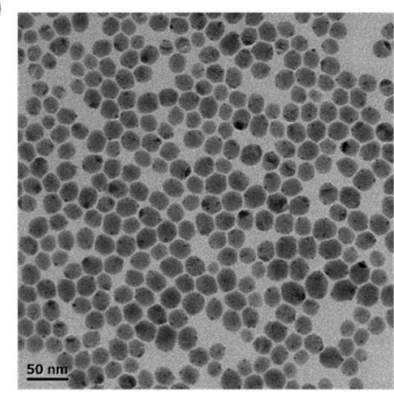

c)

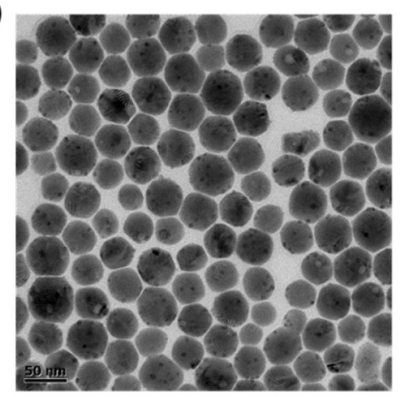

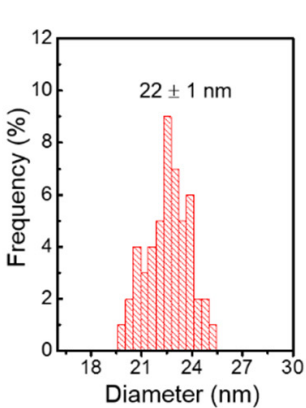

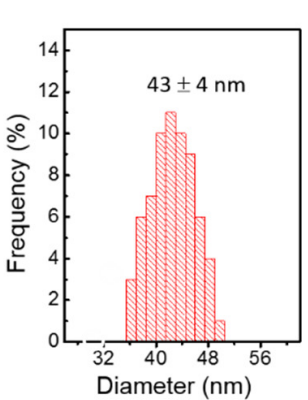

b)
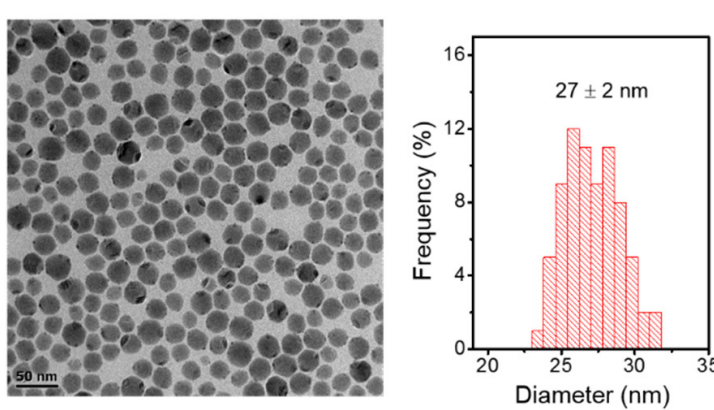

d)
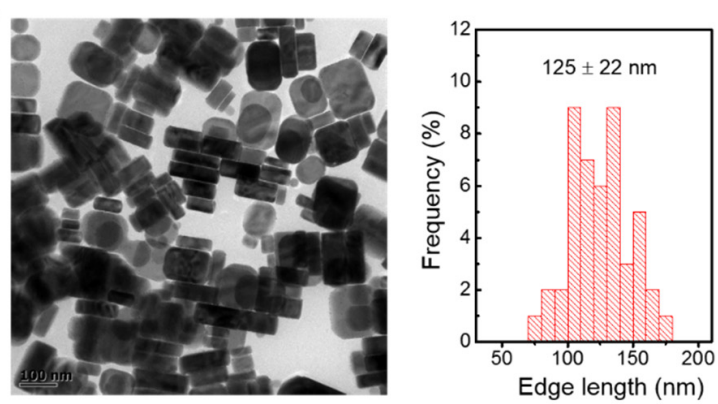

Figure S9. TEM image and histograms showing the size distribution of as-prepared $\mathrm{Cs}_{3} \mathrm{Cu}_{2} \mathrm{Br}_{5}$ nanocrystals at a) $70^{\circ} \mathrm{C}$, b) $100^{\circ} \mathrm{C}$, c) $130^{\circ} \mathrm{C}$, and d) $160^{\circ} \mathrm{C}$.

\section{Effect of reaction temperature}

The effect of reaction temperature was also examined to further optimize the synthesis conditions (to seek further insight into the role of the ligands during nanocrystal synthesis).

Figure $\mathbf{S 8}$ shows the absorption spectra of $\mathrm{Cs}_{3} \mathrm{Cu}_{2} \mathrm{Br}_{5}$ nanocrystals synthesized using $\mathrm{OA}$ and OLA as ligands at different temperatures of $70-160^{\circ} \mathrm{C} .:$ note that reaction temperatures lower than $70^{\circ} \mathrm{C}$ were not suitable for obtaining nanocrystals. The nanocrystals obtained at $70^{\circ} \mathrm{C}$, $100^{\circ} \mathrm{C}, 130^{\circ} \mathrm{C}$, and $160^{\circ} \mathrm{C}$ showed absorption peaks centered at $270,280,285$, and $287 \mathrm{~nm}$, respectively (Figure S8). As presented in Figure S9a-c, the nanocrystals had a nanosphere morphology in the temperature range of $70-130^{\circ} \mathrm{C}$, wherein the diameter of the nanospheres increased with increasing temperature. The diameters of the nanospheres obtained at $100^{\circ} \mathrm{C}$ and $130^{\circ} \mathrm{C}$ were $27 \pm 2$ and $43 \pm 4 \mathrm{~nm}$, respectively. Further increasing the temperature up to $160^{\circ} \mathrm{C}$ resulted in formation of thick octagonal nanoplates with an average edge length of $125 \pm 22 \mathrm{~nm}$ and a thickness of $38 \mathrm{~nm}$ (Figure S9d). However, the edge length of the octagonal nanoplates exceeded $100 \mathrm{~nm}$, so they were unstable in solution and tended to precipitate. 


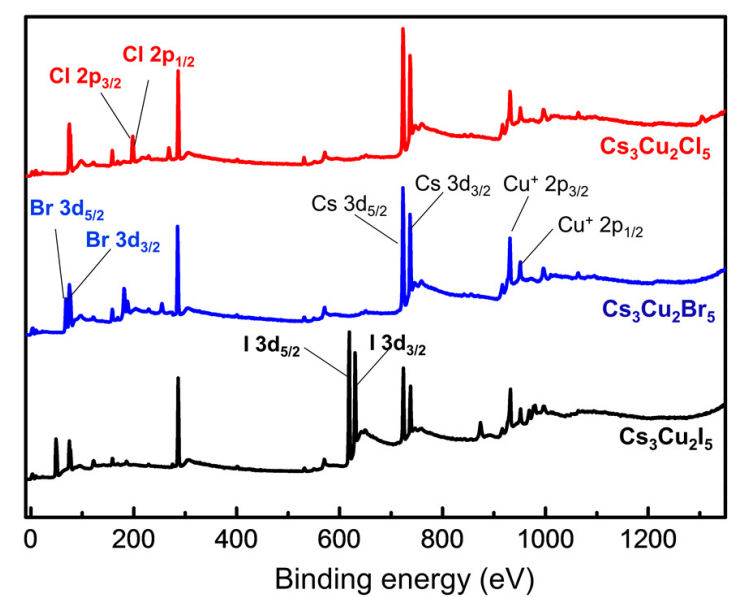

Figure S10. XPS spectra of $\mathrm{Cs}_{3} \mathrm{Cu}_{2} \mathrm{I}_{5}, \mathrm{Cs}_{3} \mathrm{Cu}_{2} \mathrm{Br}_{5}$, and $\mathrm{Cs}_{3} \mathrm{Cu}_{2} \mathrm{Cl}_{5}$ nanocrystals. 

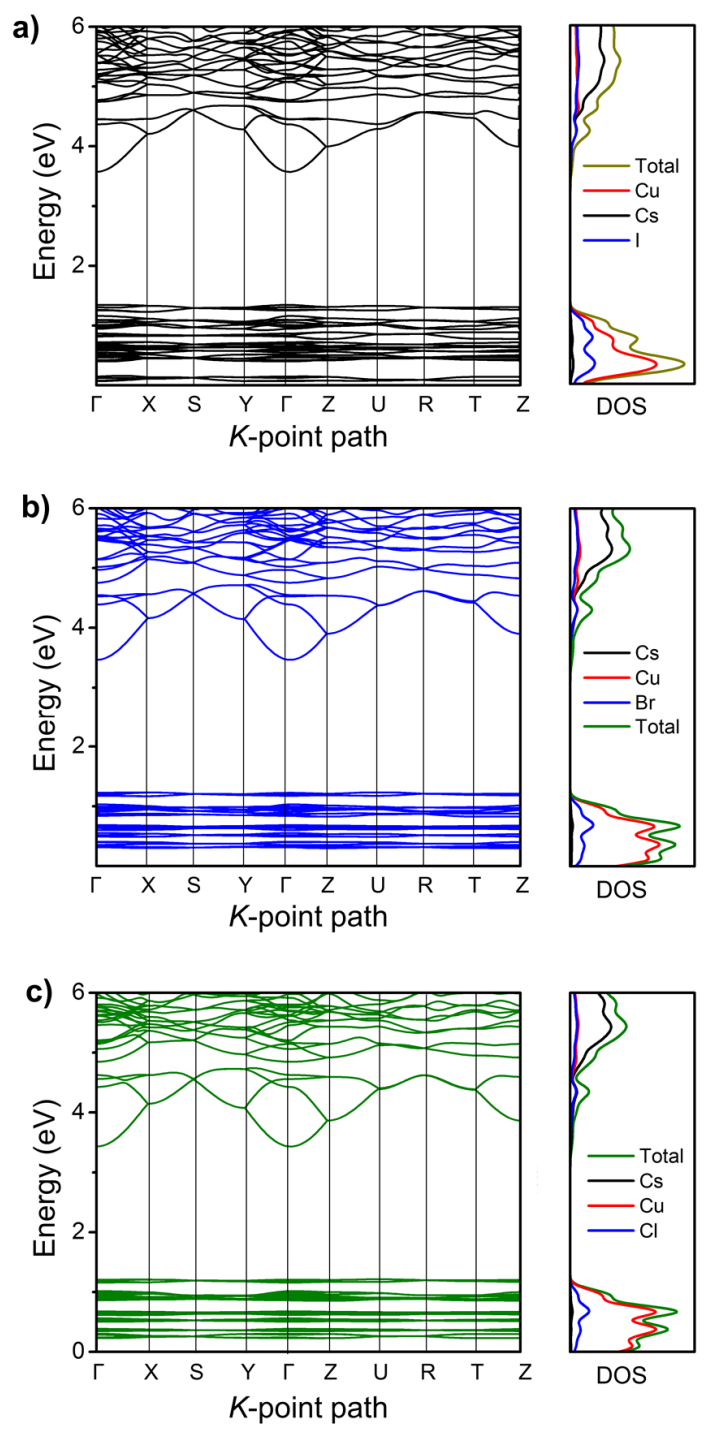

Figure S11. Electronic band structure DOSs calculated at the PBE for a) $\mathrm{Cs}_{3} \mathrm{Cu}_{2} \mathrm{I}_{5}$, b) $\mathrm{Cs}_{3} \mathrm{Cu}_{2} \mathrm{Br}_{5}$, and c) $\mathrm{Cs}_{3} \mathrm{Cu}_{2} \mathrm{Cl}_{5}$. 


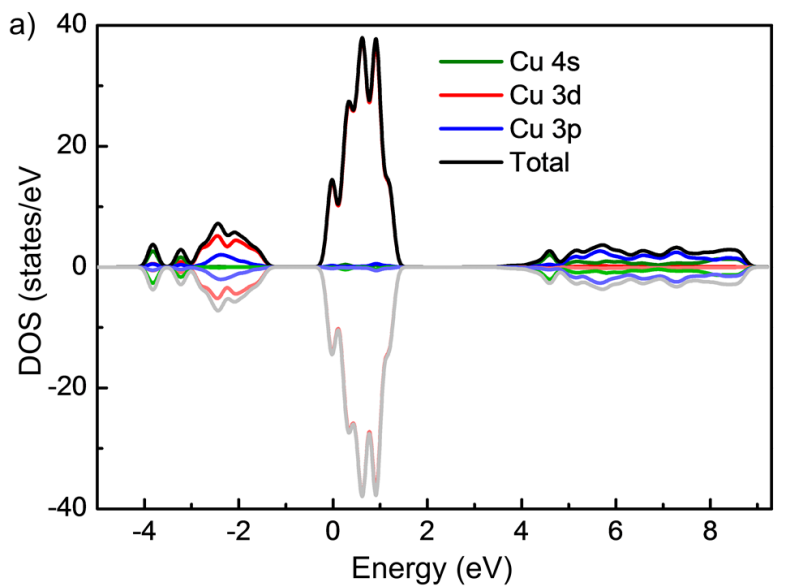

Figure S12. Detailed DOSs of $\mathrm{Cu}$ in $\mathrm{Cs}_{3} \mathrm{Cu}_{2} \mathrm{X}_{5}$. 

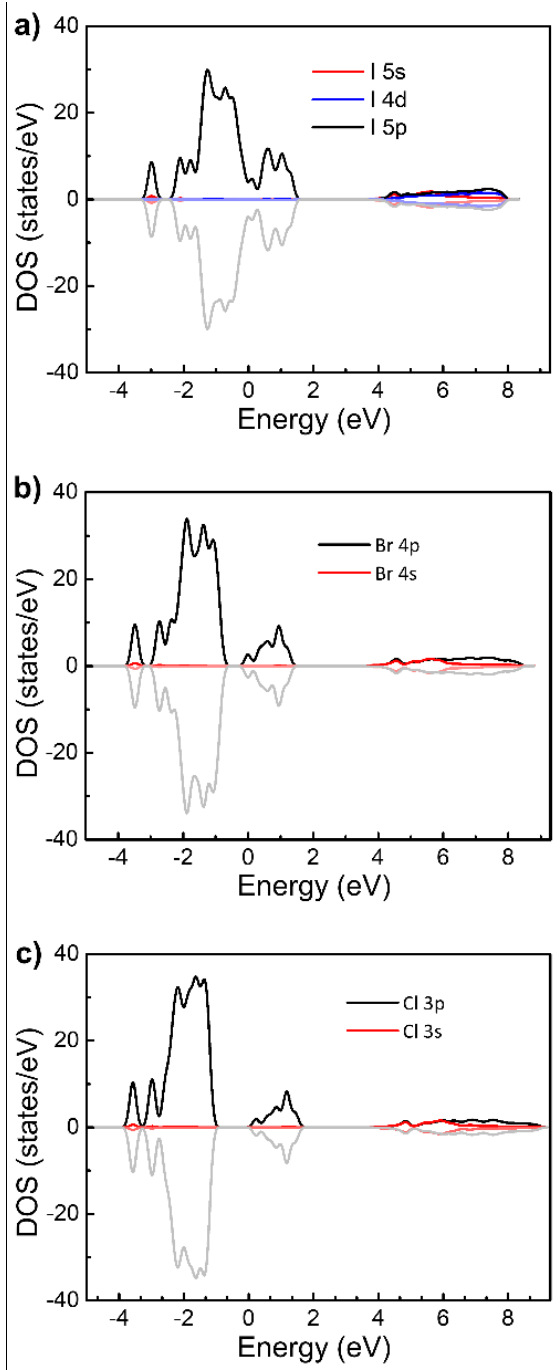

Figure S13. Detailed DOSs of a) I in $\left.\mathrm{Cs}_{3} \mathrm{Cu}_{2} \mathrm{I} 5, \mathrm{~b}\right) \mathrm{Br}$ in $\mathrm{Cs}_{3} \mathrm{Cu}_{2} \mathrm{Br}$, and c) $\mathrm{Cl}$ in $\mathrm{Cs}_{3} \mathrm{Cu}_{2} \mathrm{Cl}_{5}$.

\section{Reference}

1. Kumar, S.; Jagielski, J.; Marcato, T.; Solari, S. F.; Shih, C.-J., Understanding the ligand effects on photophysical, optical, and electroluminescent characteristics of hybrid lead halide perovskite nanocrystal solids. J. Phys. Chem. Lett. 2019, 10 (24), 7560-7567. 